\title{
Distribution of eosinophils in the gastrointestinal tract of children with no organic disease
}

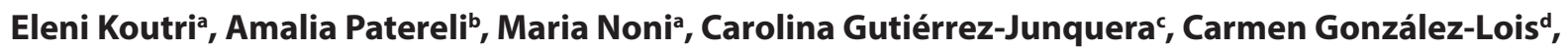 \\ Salvatore Oliva ${ }^{e}$, Carla Giordanof, Kaliopi Stefanaki ${ }^{b}$, Alexandra Papadopoulou ${ }^{a}$ \\ University of Athens, Agia Sofia Children's Hospital, Athens, Greece; University Hospital Puerta de \\ Hierro-Majadahonda, Autonomous University of Madrid, Spain; Sapienza University of Rome, Rome, Italy
}

Abstract

${ }^{a}$ Division of Gastroenterology and Hepatology, First Department of Pediatrics, University of Athens, Agia Sofia Children's Hospital, Athens, Greece (Eleni Koutri, Maria Noni, Alexandra Papadopoulou); ${ }^{b}$ Department of Pathology, Agia Sofia Children's Hospital, Athens, Greece (Amalia Patereli, Kaliopi Stefanaki); 'Pediatric Gastroenterology Unit, University Hospital Puerta de Hierro-Majadahonda, Autonomous University of Madrid, Spain (Carolina Gutiérrez-Junquera); ${ }^{\text {Pathology }}$ Department, University Hospital Puerta de Hierro Majadahonda, Autonomous University of Madrid, Spain (Carmen González-Lois); ePediatric Gastroenterology and Liver Unit, Maternal and Child Health Department, Sapienza University of Rome, Rome, Italy (Salvatore Oliva); ${ }^{\mathrm{f} D e p a r t m e n t}$ of Radiological, Oncological and Pathological Sciences, Sapienza University of Rome, Rome, Italy (Carla Giordano)

Conflict of Interest: None

Correspondence to: Alexandra Papadopoulou, Chief of the Division of Gastroenterology and Hepatology, First Department of Pediatrics, University of Athens, Agia Sofia Children's hospital, Thivon and Papadiamantopoulou, 11527, Athens, Greece, e-mail: a.papadopoulou@paidon-agiasofia.gr

Received 4 February 2020; accepted 24 May 2020; published online 22 June 2020

DOI: https://doi.org/10.20524/aog.2020.0518

\section{Introduction}

Eosinophilic gastrointestinal (GI) disorders (EGID) comprise a rare group of chronic inflammatory disorders of the GI tract attracting growing interest. Clinically, they are characterized by various symptoms, depending on the involved segment of the GI tract, and histologically, by dense eosinophilic infiltration in the absence of an identifiable secondary cause [1]. However, the eosinophilic density of GI tract mucosa of healthy children, which would provide baseline data for the diagnosis of EGIDs, has been poorly defined [2-5]. Inflammatory bowel disease has been reported to be associated with increased eosinophilic infiltration of the childhood GI tract [6], while the association of functional GI disorders (FGIDs) with increased eosinophil (eos) density of the child's GI tract is controversial: some studies report increased eosinophilic infiltration of the GI tissue mucosa in children with FGIDs [7], whereas others do not [8]. Furthermore, the geographical variations in the distribution or density of GI tissue eos in children with no organic diseases have been poorly defined. 
The aim of our study was to assess the eos distribution and density in the GI tract in a pediatric population undergoing endoscopy after an extensive workup, with no subsequent diagnosis of an organic disease.

\section{Patients and methods}

Children who underwent GI endoscopies at 3 referral pediatric gastroenterology units (Athens, Madrid and Rome) between January 2012 and June 2018 were included in this study if they had a normal workup prior to endoscopy, normal macroscopic appearance during endoscopy, and no evidence of organic disease, either at the time of endoscopy or at least 1 year after. The workup to exclude organic diseases included, but was not limited to, the following: full blood count, C-reactive protein, erythrocyte sedimentation rate, renal and liver function tests, serum amylase, total immunoglobulin A (IgA), tissue transglutaminase IgA antibodies, and urinalysis, while for patients undergoing colonoscopy the workup also included stool tests for viruses and parasites, and stool cultures for bacteria. The exclusion of an organic disease was based on history, clinical examination, extensive work up, normal macroscopic findings during the GI endoscopy and non-specific histological findings in the GI biopsies.

Patients diagnosed with FGIDs were not excluded and were compared to those without the diagnosis of FGIDs. Demographic and clinical data, including age, sex, primary indication for endoscopy and the final diagnosis, were also recorded.

After the selection of the patients to be included in the study, we retrieved the hematoxylin and eosin histological slides of each patient to count the eos. The criteria and the procedure for the histology assessment were discussed and agreed by all of the pathologists before the start of the study. The slides were reviewed by a single pathologist not aware of the prior histology report, while a subset of data was subsequently compared and reviewed by a senior pathologist (KS). An eos was counted when 2 nuclei were recognized. All biopsies were assessed to select the area with highest eos density and at least 5 high power fields (hpf) were subsequently evaluated to determine the peak density of eos in each GI segment. Quantification of eos was performed manually. Peak eosinophilic counts were expressed $/ \mathrm{hpf}$ and $/ \mathrm{mm}^{2}$. The areas of hpfs of the microscopes used by different centers were $0.196 \mathrm{~mm}^{2}$ (Athens) and $0.306 \mathrm{~mm}^{2}$ (Madrid and Rome). In order to have homogeneous histology reports we recalculated the eos counts to express all of them per hpfs of both sizes $\left(0.196 \mathrm{~mm}^{2}\right.$ and $0.306 \mathrm{~mm}^{2}$ respectively), but also per $\mathrm{mm}^{2}$ (eosinophil density) using the following formula: eos $/ \mathrm{mm}^{2}=\operatorname{eos} / \mathrm{hpf} \times 1 /$ area of microscope $\mathrm{hpf}$ in $\mathrm{mm}^{2}$. Other histological abnormalities, such as eosinophilic surface layering, eosinophilic degranulation, basal zone hyperplasia, lamina propria fibrosis, increased intraepithelial eos, dilated intercellular spaces, and eosinophilic cryptitis/pititis, as defined by Collins et al, were also reported [9].

The study was first approved by the Institutional Review Board (IRB) of the "Agia Sofia" Athens Children's hospital. Subsequently, ethical approval was obtained for the co-participating centers by their local IRBs.

\section{Statistical analysis}

Descriptive analysis was performed for all variables. Categorical data were expressed as absolute number and proportions (\%), while continuous variables were reported as the median and interquartile range (IQR), since the distribution was not normal. Continuous data were tested for normality using statistical tests (Kolmogorov-Smirnoff test) and graphical methods (histogram, Q-Q plot). Because all data were nonparametric, Mann-Whitney and Kruskal-Wallis tests were used in the comparative analyses. Boxplots were also used for the graphical presentation of continuous data in figures. Results were presented in eos $/ \mathrm{mm}^{2}$, as in recent studies in the literature. However, the median density of eos/hpf was also calculated for a better comparison with other studies. All statistical analyses were performed using the statistical package PSAW Statistics v23 (SPSS, Inc., Chicago, IL, USA). Statistical significance at $\mathrm{P}<0.05$ was assumed.

\section{Results}

A total of 155 patients (73 boys) who underwent GI endoscopies with 595 biopsies between January 2012 and June 2018 were included in the study. Of these, 111 patients (48 boys), median (IQR) age 11 (7-13) years, underwent upper endoscopy with 333 biopsies (111 from the esophagus, 111 from the stomach and 111 from the $2^{\text {nd }} / 3^{\text {rd }}$ parts of the duodenum), while 44 (25 boys), median (IQR) age 12 (7-14) years, also underwent ileocolonoscopy with 262 biopsies (44 from the ileum, 37 from the cecum, 28 from the ascending colon, 44 from the transverse colon, 31 from the descending colon, 37 from the sigmoid colon and 41 biopsies from the rectum). With regard to the gastric biopsies, they were taken from different parts of the stomach at different centers: 76 were taken from the gastric body (Athens), 20 from the gastric fundus (Rome), and 15 from the gastric antrum (Madrid).

In total, 1012 slides were reviewed by a single pathologist in each center, as follows: 710 in Athens (AP), 152 in Madrid (CGon) and 150 in Rome (CGio). The reports on the 710/1012 slides reviewed in Athens were subsequently compared and reviewed by a senior pathologist (KS). Lamina propria was available in all of the biopsies beyond the esophagus, but in only 42 of 111 biopsies taken from the esophagus.

The most common reason for endoscopy in the total cohort was retrosternal and/or epigastric pain $(n=69 ; 44.5 \%)$, followed by abdominal pain $(n=36 ; 23.2 \%)$ and diarrhea $(\mathrm{n}=17 ; 11 \%)$, while $62(40 \%)$ patients experienced more than one symptom. The median (IQR) follow up of the patients was 23.35 (15.1-31.5) months.

With regard to the eosinophilic distribution across the GI tract, a gradual increase in GI tissue eos was observed in the proximal segments of the GI tract, from the esophagus to the ileum, while in the colon, a gradual decline was observed from the cecum to the rectum. The median (IQR) of peak counts of eos/hpf corresponding to areas of $0.196 \mathrm{~mm}^{2}$ and $0.306 \mathrm{~mm}^{2}$, as well as the count corresponding to $\mathrm{mm}^{2}$ in each segment of the GI tract for the whole cohort, are shown in Fig. 1-3. 
Interestingly, significant differences among the 3 centers were observed in the eosinophilic counts of all segments of the GI tract (Table 1), with the Athens center showing greater eos counts in all of the segments except the esophagus. Seasonal variation as a potential reason for the differences found was excluded, as the timing of the performed endoscopies varied within the year in all centers. The role, however, of other potential reasons, such as diet, drugs or history of atopy were not recorded in this retrospective study; thus, the potential impact of the above factors on geographical variation needs further investigation.

With regard to the evaluation of any associations of FGIDs with GI tissue eosinophilia, for the sake of homogeneity, we applied the same criteria (Rome IV criteria) [10] that have been valid since May 2016 for the diagnosis of FGID to all the patients. As recent studies [11] reported that using Rome III led to underestimation [12] of the diagnosis of FGIDs associated with abdominal pain, compared to the Rome IV [10] criteria,

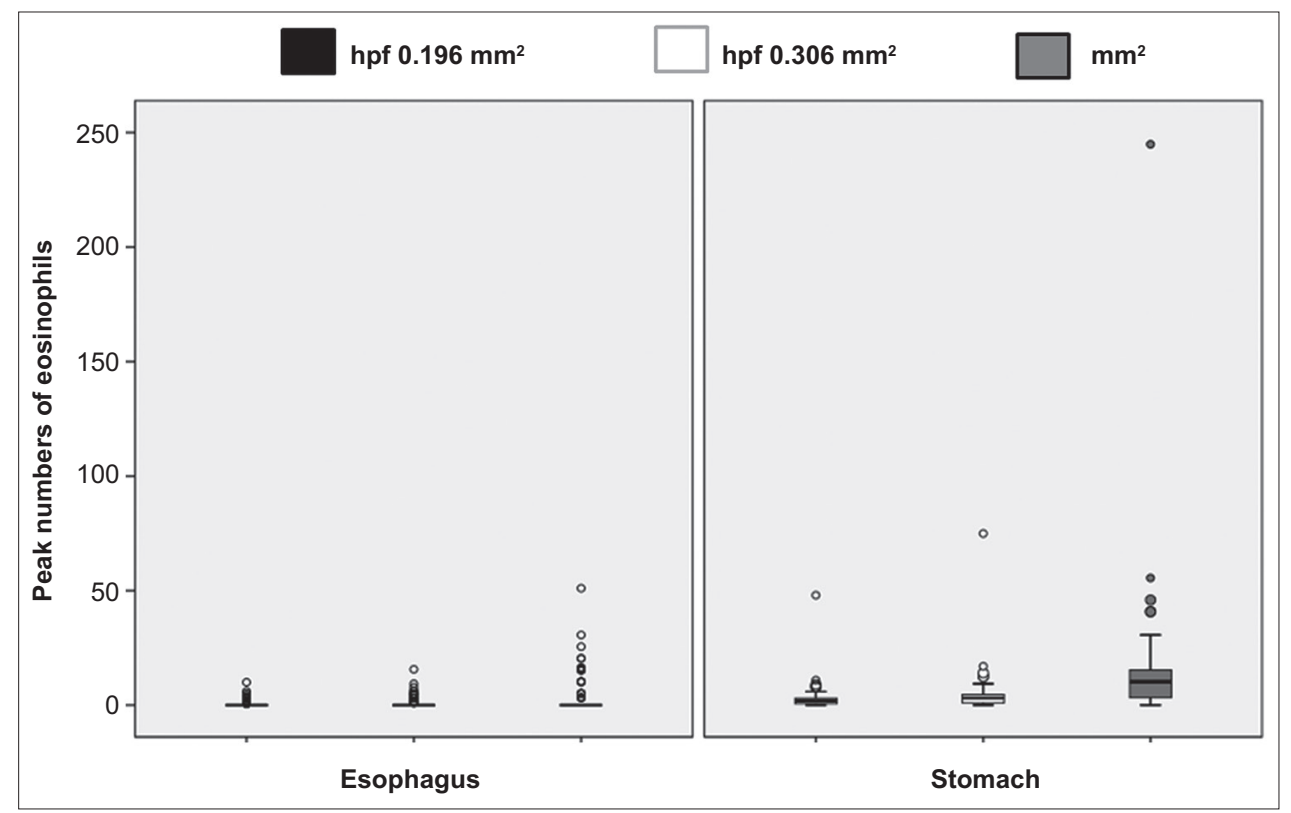

Figure 1 Peak numbers of eosinophils in the esophageal and gastric biopsies in the whole cohort of children. Median (interquartile range) of peak counts of eosinophils /high power field $0.196 \mathrm{~mm}^{2}$ and $0.306 \mathrm{~mm}^{2}$, and $/ \mathrm{mm}^{2}$ in the esophagus (111 biopsies) and the stomach (111 biopsies) were as follows: esophagus: $0(0-0), 0(0-0)$ and $0(0-0)$, respectively; stomach: $2(0.6-3.0), 3.1(1.0-4.7)$ and 10.2 (3.3-15.3), respectively

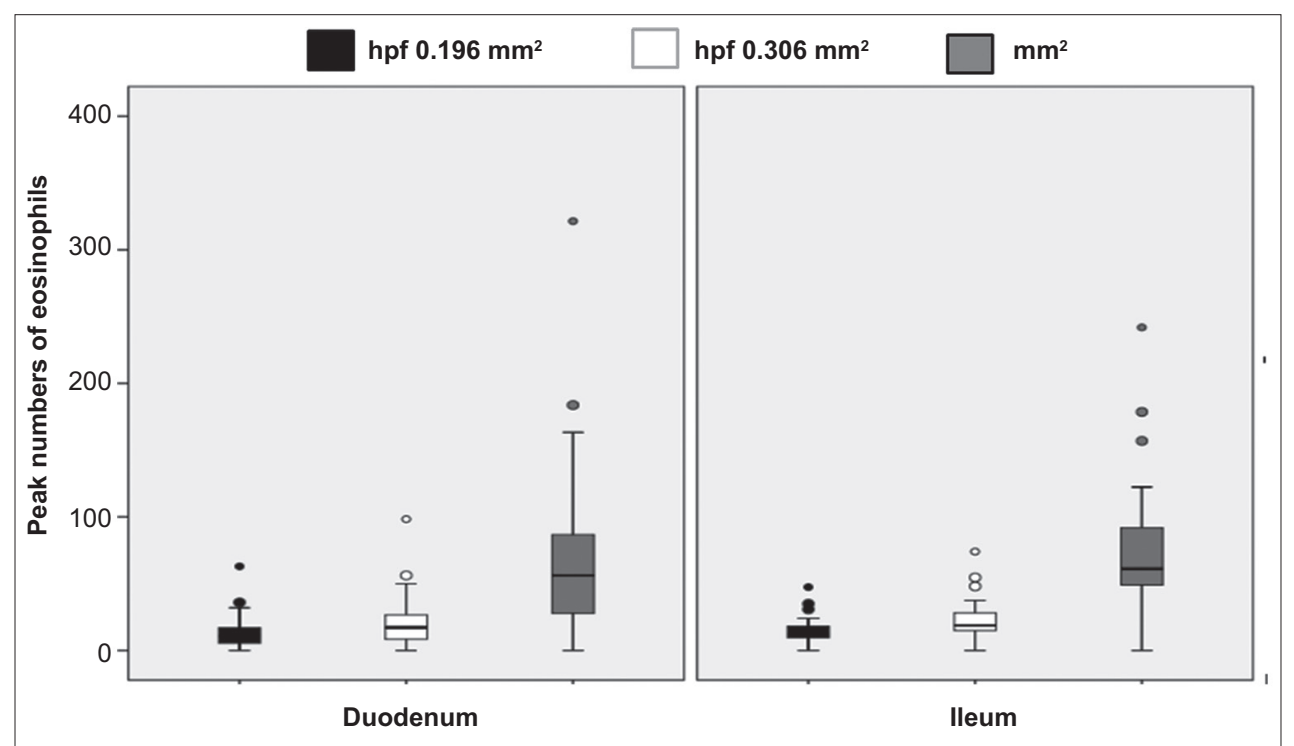

Figure 2 Peak numbers of eosinophils in the duodenal and ileal biopsies in the whole cohort of children. Median (interquartile range) of peak counts of eosinophils /high power field $0.196 \mathrm{~mm}^{2}$ and $0.306 \mathrm{~mm}^{2}$, and $/ \mathrm{mm}^{2}$ in the duodenum (111 biopsies) and ileum (44 biopsies) were as follows: duodenum: 11.0 (5.1-17.0), 17.2 (8.0-26.5) and 56.1 (26.1-86.7), respectively; ileum: 12.0 (9.6-18.0), 18.7 (15.0-28.1) and 61.2 (49.0-91.8), respectively 


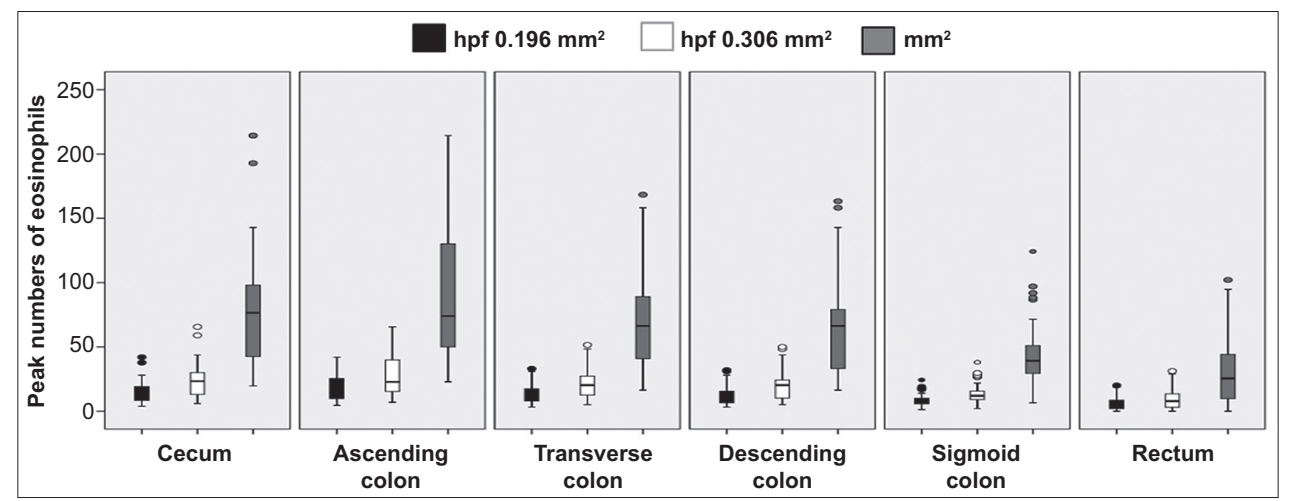

Figure 3 Peak numbers of eosinophils in the colonic biopsies in the whole cohort of children. Median (interquartile range) of peak counts of eosinophils /high power field $0.196 \mathrm{~mm}^{2}$ and $0.306 \mathrm{~mm}^{2}$, and $/ \mathrm{mm}^{2}$ in the cecum (37 biopsies), ascending colon ( 28 biopsies), transverse colon (44 biopsies), descending colon (31 biopsies), sigmoid colon (37 biopsies) and rectum (41 biopsies) were as follows: cecum: 15.0 (8.0-19.5), 23.4 (12.5$30.5)$ and 76.5 (40.9-99.7), respectively; ascending colon: 14.5 (9.7-25.8), 22.8 (15.2-40.2) and 73.9 (49.5-131.4), respectively; transverse colon: 13.0 (8.0-17.9), 20.3 (12.5-28.0) and 66.3 (40.8-91.5), respectively; descending colon: 13.0 (6.0-16.0), 20.3 (9.4-24.9) and 66.3 (30.6-81.6), respectively; sigmoid colon: 7.6 (5.4-10.0), 12.0 (8.5-15.6) and 39.2 (27.8-51.0), respectively; rectum: 5.0 (1.9-8.9), 7.8 (3.0-14.0) and 25.5 (9.8-45.8), respectively

we decided to apply the same criteria (Rome IV criteria) to all of our patients, to avoid any bias by missing FGID diagnoses. Interestingly, we found that the Rome IV criteria were fulfilled for the diagnosis of FGID by 73 children (37 boys), median (IQR) age 13 (9-14) years, while the Rome III criteria [12] were satisfied by only 39 children (20 boys), median (range) age 13 (3-17) years.

$\mathrm{FGID}^{+}$patients were found to be older than FGID: their median (IQR) age was 13 (9-14) vs. 10 (6.5-12) years, respectively $(\mathrm{P}<0.001)$, while no difference was found with regard to sex $(\mathrm{P}=0.489)$. The difference in age in $\mathrm{FGID}^{+}$children was found in both children undergoing upper endoscopy-median (IQR) age 13 (10-14) years vs. 10 (6-12), respectively $(\mathrm{P}=0.002)$-and in those undergoing lower endoscopy: median (IQR) age 13 (8-14) years vs. $10(6.5-12)$ years, respectively $(\mathrm{P}<0.001)$.

The main reason for endoscopy in $\mathrm{FGID}^{+}$compared to FGID children was retrosternal and/or epigastric pain (26/73 vs. $43 / 83$, respectively; $\mathrm{P}=0.035$ ), followed by vomiting ( $22 / 73$ vs. $14 / 82$, respectively; $\mathrm{P}=0.55)$, abdominal pain $(21 / 73$ vs. $15 / 82$, respectively; $\mathrm{P}=0.123$ ), and diarrhea (15 vs. 2, respectively; $\mathrm{P}<0.001)$. It should be noted, however, that $40 / 73(54.8 \%)$ of $\mathrm{FGID}^{+}$and 22/82 (26.8\%) of FGID patients experienced a combination of symptoms. The FGID diagnoses based on Rome IV criteria were as follows: functional dyspepsia (FD) in 28 (18.1\%) children; irritable bowel syndrome (IBS) with predominant diarrhea in 15 (9.7\%); IBS with predominant constipation in 8 (5.2\%); IBS unclassified in 4 (2.6\%); functional abdominal pain unspecified in 10 (6.4\%); and functional vomiting in 8 (5.2\%).

No significant differences in the eosinophilic distribution or the eosinophilic density of the GI tissue were found between children with and those without FGIDs in any of the segments of the GI tract (Table 2). We did find, however, elevated intraepithelial eos in a greater number of children in the $\mathrm{FGID}^{+}$ group ( $8.1 \%$ vs. $2.2 \%$, respectively; $\mathrm{P}=0.003$ ) and the same was true for eosinophilic cryptitis $(20.1 \%$ vs. $7.5 \%$, respectively; $\mathrm{P}<0.001$ ), while pititis was absent in all of the gastric biopsies. Mild eosinophilic degranulation toward the edge of the tissue biopsy sample occurred in $37 \%$ of all biopsies, with a similar incidence in both groups (39.6\% in $\mathrm{FGID}^{+}$vs. $35.8 \%$ in FGID; $\mathrm{P}=0.344$ ) and the same was true for dilated intercellular spaces $(6.4 \%$ vs. $1.6 \%$, respectively; $\mathrm{P}=0.178$ ). Eosinophilic surface layering was absent in all esophageal biopsies, while mild lamina propria fibrosis was rare, as it was found in only 3 (2 FGID ${ }^{+}$and 1 FGID) of the 42 patients whose biopsies included lamina propria.

\section{Discussion}

This study assessed the normal eosinophilic density and distribution, as well as other histopathologic parameters, in the mucosa of the GI tract in children who underwent endoscopic procedures after an extensive workup due to GI symptoms, without subsequent diagnosis of an organic disease, either at the time of endoscopy or within 1 year after.

The main value of this study is that it provides normative values of eos in the "healthy" childhood GI mucosa that will be useful in the diagnosis of childhood EGIDs beyond the esophagus which in the absence of baseline data, is ofter a challenging issue, as it relys on the pathologist's subjective judgment.

In accordance with previous studies, the distribution of the eos in our study showed a gradual increase across the proximal segments of the GI tract, from the esophagus to the ileum, followed by a subsequent gradual decrease from the first segments of the colon to the rectum $[3,4]$. The range of eos/hpf in esophageal biopsies in our study was consistent with the findings of previous studies that reported up to $6 \mathrm{eos} / \mathrm{hpf}$ in healthy esophageal mucosa of children between $7-15$ years of age $[13,14]$.

With regard to the eosinophilic infiltration of different parts of the gastric mucosa, the reports are conflicting. Some studies have shown no differences in the eosinophilic infiltration of the gastric antrum compared to the gastric body [3], while others reported a greater eosinophilic infiltration of the gastric antrum (but not of the gastric body) in children with functional abdominal pain disorders compared to healthy controls [6]. The differences in eosinophilic counts found between the different parts of the stomach in our study were not related to 
Table 1 Peak eosinophil counts in the epithelium of different segments of the gastrointestinal (GI) tract amongst participating centers

\begin{tabular}{|c|c|c|c|c|}
\hline GI segment & Greece & Spain & Italy & $P$ value \\
\hline \multirow{3}{*}{$\begin{array}{l}\text { Total } \mathrm{N}=595 \text { biopsies } \\
\text { Total } \mathrm{N}=1012 \text { slides }\end{array}$} & $\mathrm{N}=351$ biopsies & N=99 biopsies; & $\mathrm{N}=145$ biopsies; & \\
\hline & $\mathrm{N}=710$ slides & $\mathrm{N}=154$ slides & $\mathrm{N}=150$ slides & \\
\hline & Median (IQR) & Median (IQR) & Median (IQR) & \\
\hline \multirow[t]{2}{*}{ Esophagus } & $\mathrm{N}=72$ biopsies & $\mathrm{N}=15$ biopsies & $\mathrm{N}=24$ biopsies & \\
\hline & $\mathrm{N}=154$ slides & $\mathrm{N}=48$ slides & $\mathrm{N}=24$ slides & \\
\hline Eos/hpf $0.196 \mathrm{~mm}^{2}$ & $0.0(0.0-0.0)$ & $0.0(0.0-0.6)$ & $0.0(0.0-0.0)$ & 0.050 \\
\hline Eos/hpf $0.306 \mathrm{~mm}^{2}$ & $0.0(0.0-0.0)$ & $0.0(0.0-1.0)$ & $0.0(0.0-0.0)$ & 0.050 \\
\hline $\mathrm{Eos} / \mathrm{mm}^{2}$ & $0.0(0.0-0.0)$ & $0.0(0.0-3.3)$ & $0.0(0.0-0.0)$ & 0.050 \\
\hline Stomach & $\begin{array}{c}\mathrm{N}=76 \text { biopsies } \\
\text { (Body) }\end{array}$ & $\begin{array}{c}\mathrm{N}=15 \text { biopsies } \\
\text { (Antrum) }\end{array}$ & $\begin{array}{c}\mathrm{N}=20 \text { biopsies } \\
\quad \text { (Fundus) }\end{array}$ & \\
\hline Eos/hpf $0.196 \mathrm{~mm}^{2}$ & $\mathrm{~N}=154$ slides & $\mathrm{N}=24$ slides & $\mathrm{N}=20$ slides & \\
\hline Eos/hpf $0.306 \mathrm{~mm}^{2}$ & $2.0(1.0-4.0)$ & $0.6(0.0-1.3)$ & $0.6(0.0-1.1)$ & 0.000 \\
\hline \multirow[t]{2}{*}{$\mathrm{Eos} / \mathrm{mm}^{2}$} & $3.1(1.6-6.2)$ & $1.0(0.0-2.0)$ & $1.0(0.0-1.8)$ & 0.000 \\
\hline & $10.2(5.1-20.4)$ & $3.3(0.0-6.5)$ & $3.3(0.0-5.7)$ & 0.000 \\
\hline Duodenum & $\begin{array}{c}\mathrm{N}=76 \text { biopsies } \\
\mathrm{N}=154 \text { slides }\end{array}$ & $\begin{array}{c}\mathrm{N}=15 \text { biopsies } \\
\mathrm{N}=25 \text { slides }\end{array}$ & $\begin{array}{c}\mathrm{N}=20 \text { biopsies } \\
\mathrm{N}=20 \text { slides }\end{array}$ & \\
\hline Eos/hpf $0.196 \mathrm{~mm}^{2}$ & $15.0(11.0-18.8)$ & $3.2(1.9-5.1)$ & $5.1(3.4-6.2)$ & 0.000 \\
\hline Eos/hpf $0.306 \mathrm{~mm}^{2}$ & $23.4(17.2-29.3)$ & $5.0(3.0-8.0)$ & $8.0(5.3-9.8)$ & 0.000 \\
\hline $\mathrm{Eos} / \mathrm{mm}^{2}$ & $76.5(56.1-95.7)$ & $16.3(9.8-26.1)$ & $26.1(17.2-31.9)$ & 0.000 \\
\hline Ileum & $\begin{array}{c}\mathrm{N}=20 \text { biopsies } \\
\mathrm{N}=42 \text { slides }\end{array}$ & $\begin{array}{c}\mathrm{N}=6 \text { biopsies } \\
\mathrm{N}=6 \text { slides }\end{array}$ & $\begin{array}{c}\mathrm{N}=18 \text { biopsies } \\
\mathrm{N}=18 \text { slides }\end{array}$ & \\
\hline Eos/hpf $0.196 \mathrm{~mm}^{2}$ & $15.0(12.0-19.5)$ & $9.6(3.5-12.2)$ & $9.6(4.3-14.1)$ & 0.001 \\
\hline Eos/hpf $0.306 \mathrm{~mm}^{2}$ & $23.4(18.7-30.4)$ & $15.0(5.5-19.0)$ & $15.0(6.8-22.0)$ & 0.001 \\
\hline $\mathrm{Eos} / \mathrm{mm}^{2}$ & $76.5(61.2-99.5)$ & $49.0(17.9-62.9)$ & $49.0(22.1-71.9)$ & 0.001 \\
\hline Cecum & $\begin{array}{c}\mathrm{N}=13 \text { biopsies } \\
\mathrm{N}=24 \text { slides }\end{array}$ & $\begin{array}{c}\mathrm{N}=8 \text { biopsies } \\
\mathrm{N}=8 \text { slides }\end{array}$ & $\begin{array}{c}\mathrm{N}=16 \text { biopsies } \\
\mathrm{N}=17 \text { slides }\end{array}$ & \\
\hline Eos/hpf $0.196 \mathrm{~mm}^{2}$ & $18.0(15.3-26.5)$ & $7.1(4.5-10.6)$ & $14.7(8.9-19.5)$ & 0.002 \\
\hline Eos/hpf $0.306 \mathrm{~mm}^{2}$ & $28.1(23.8-41.4)$ & $11.0(7.0-16.5)$ & $23.0(14.0-30.5)$ & 0.002 \\
\hline $\mathrm{Eos} / \mathrm{mm}^{2}$ & $91.8(77.8-135.2)$ & $35.9(22.9-53.9)$ & $75.2(45.8-99.7)$ & 0.002 \\
\hline Ascending colon & $\begin{array}{c}\mathrm{N}=21 \text { biopsies } \\
\mathrm{N}=42 \text { slides }\end{array}$ & $\begin{array}{c}\mathrm{N}=7 \text { biopsies } \\
\mathrm{N}=7 \text { slides }\end{array}$ & ---- & \\
\hline Eos/hpf $0.196 \mathrm{~mm}^{2}$ & $19.0(13.5-27.5)$ & $7.7(4.5-9.6)$ & ----- & 0.000 \\
\hline Eos/hpf $0.306 \mathrm{~mm}^{2}$ & $29.7(21.1-42.9)$ & $12.0(7.0-15.0)$ & ----- & 0.000 \\
\hline Eos $/ \mathrm{mm}^{2}$ & $96.9(68.9-140.3)$ & $39.2(22.9-49.0)$ & & 0.000 \\
\hline Transverse colon & $\begin{array}{c}\mathrm{N}=20 \text { biopsies } \\
\mathrm{N}=38 \text { slides }\end{array}$ & $\begin{array}{c}\mathrm{N}=8 \text { biopsies } \\
\mathrm{N}=8 \text { slides }\end{array}$ & $\begin{array}{c}\mathrm{N}=16 \text { biopsies } \\
\mathrm{N}=17 \text { slides }\end{array}$ & \\
\hline Eos/hpf $0.196 \mathrm{~mm}^{2}$ & $15.5(12.3-22.8)$ & $7.1(3.7-8.9)$ & $12.2(5.8-20.5)$ & 0.004 \\
\hline Eos/hpf $0.306 \mathrm{~mm}^{2}$ & $24.2(19.1-35.5)$ & $11.0(5.8-14.0)$ & $19.0(9.0-32.0)$ & 0.004 \\
\hline $\mathrm{Eos} / \mathrm{mm}^{2}$ & $79.1(62.5-116.1)$ & $35.9(18.8-45.8)$ & $62.1(29.4-104.6)$ & 0.004 \\
\hline Descending colon & $\begin{array}{c}\mathrm{N}=22 \text { biopsies } \\
\mathrm{N}=44 \text { slides }\end{array}$ & $\begin{array}{c}\mathrm{N}=9 \text { biopsies } \\
\mathrm{N}=9 \text { slides }\end{array}$ & ----- & \\
\hline Eos/hpf $0.196 \mathrm{~mm}^{2}$ & $14.0(10.8-20.5)$ & $5.1(4.2-7.7)$ & ----- & 0.000 \\
\hline Eos/hpf $0.306 \mathrm{~mm}^{2}$ & $21.9(16.8-32.0)$ & $8.0(6.5-12.0)$ & ----- & 0.000 \\
\hline $\mathrm{Eos} / \mathrm{mm}^{2}$ & $71.4(54.8-104.6)$ & $26.1(21.2-39.2)$ & & 0.000 \\
\hline Sigmoid & $\mathrm{N}=13$ biopsies & $\mathrm{N}=7$ biopsies & $\mathrm{N}=17$ biopsies & \\
\hline Eos/hpf $0.196 \mathrm{~mm}^{2}$ & $\mathrm{~N}=26$ slides & $\mathrm{N}=8$ slides & $\mathrm{N}=17$ slides & \\
\hline Eos/hpf $0.306 \mathrm{~mm}^{2}$ & $10.0(7.5-15.5)$ & $7.1(3.2-9.6)$ & $5.8(4.2-8.9)$ & 0.018 \\
\hline \multirow{2}{*}{ Eos $/ \mathrm{mm}^{2}$} & $15.6(11.7-24.2)$ & $11.0(5.0-15.0)$ & $9.0(6.5-14.0)$ & 0.018 \\
\hline & $51.0(38.2-79.1)$ & $35.9(16.3-49.0)$ & $29.4(21.2-44.8)$ & 0.018 \\
\hline Rectum & $\begin{array}{c}\mathrm{N}=18 \text { biopsies } \\
\mathrm{N}=32 \text { slides }\end{array}$ & $\begin{array}{c}\mathrm{N}=9 \text { biopsies } \\
\mathrm{N}=9 \text { slides }\end{array}$ & $\begin{array}{c}\mathrm{N}=14 \text { biopsies } \\
\mathrm{N}=17 \text { slides }\end{array}$ & \\
\hline Eos/hpf $0.196 \mathrm{~mm}^{2}$ & $6.0(5.0-9.8)$ & $5.1(1.9-7.4)$ & $1.6(0.5-8.2)$ & 0.018 \\
\hline Eos/hpf $0.306 \mathrm{~mm}^{2}$ & $9.4(7.8-15.2)$ & $8.0(3.0-11.5)$ & $2.5(0.8-12.8)$ & 0.018 \\
\hline $\mathrm{Eos} / \mathrm{mm}^{2}$ & $30.6(25.5-49.7)$ & $26.1(9.8-37.6)$ & $8.2(2.5-41.7)$ & 0.018 \\
\hline
\end{tabular}

Eos/hpf, Eosinophils per high power field; IQR, interquartile range 
Table 2 Eosinophilic counts in children with or without FGIDs

\begin{tabular}{|c|c|c|c|}
\hline Segment of GI tract & FGID $^{+} \mathrm{N}=293$ biopsies & FGID $\mathrm{N}=302$ biopsies & $P$ value \\
\hline $\mathrm{N}=595$ biopsies & Median (IQR) & Median (IQR) & \\
\hline Esophagus & $\mathrm{N}=47$ biopsies & $\mathrm{N}=64$ biopsies & \\
\hline Eos/hpf $0.196 \mathrm{~mm}^{2}$ & $0.0(0.0-0.0)$ & $0.0(0.0-0.0)$ & 0.756 \\
\hline Eos/hpf $0.306 \mathrm{~mm}^{2}$ & $0.0(0.0-0.0)$ & $0.0(0.0-0.0)$ & 0.756 \\
\hline $\mathrm{Eos} / \mathrm{mm}^{2}$ & $0.0(0.0-0.0)$ & $0.0(0.0-0.0)$ & 0.756 \\
\hline Stomach & $\mathrm{N}=47$ biopsies & $\mathrm{N}=64$ biopsies & \\
\hline Eos/hpf $0.196 \mathrm{~mm}^{2}$ & $1.3(0.6-4.0)$ & $2.0(1.0-3.0)$ & 0.725 \\
\hline Eos/hpf $0.306 \mathrm{~mm}^{2}$ & $2.0(1.0-6.2)$ & $3.1(1.6-4.7)$ & 0.725 \\
\hline $\mathrm{Eos} / \mathrm{mm}^{2}$ & $6.5(3.3-20.4)$ & $10.2(5.1-15.3)$ & 0.807 \\
\hline Duodenum & $\mathrm{N}=48$ biopsies & $\mathrm{N}=63$ biopsies & \\
\hline Eos/hpf $0.196 \mathrm{~mm}^{2}$ & $6.4(3.8-21.0)$ & $12.0(6.3-15.0)$ & 0.931 \\
\hline Eos/hpf $0.306 \mathrm{~mm}^{2}$ & $10.0(6.0-32.8)$ & $18.7(9.8-23.4)$ & 0.931 \\
\hline $\mathrm{Eos} / \mathrm{mm}^{2}$ & $32.7(19.6-107.1)$ & $61.2(31.9-76.5)$ & 0.931 \\
\hline Ileum & $\mathrm{N}=26$ biopsies & $\mathrm{N}=18$ biopsies & \\
\hline Eos/hpf $0.196 \mathrm{~mm}^{2}$ & $13.5(5.8-119.9)$ & $12.0(9.6-15.0)$ & 0.400 \\
\hline Eos/hpf $0.306 \mathrm{~mm}^{2}$ & $21.0(9.0-31.1)$ & $18.7(15.0-23.4)$ & 0.400 \\
\hline $\mathrm{Eos} / \mathrm{mm}^{2}$ & $68.6(29.4-101.7)$ & $61.2(49.0-76.5)$ & 0.400 \\
\hline Cecum & $\mathrm{N}=21$ biopsies & $\mathrm{N}=16$ biopsies & \\
\hline Eos/hpf $0.196 \mathrm{~mm}^{2}$ & $12.2(7.2-21.5)$ & $15.4(10.2-18.0)$ & 0.772 \\
\hline Eos/hpf $0.306 \mathrm{~mm}^{2}$ & $19.0(11.3-33.6)$ & $24.0(15.9-28.1)$ & 0.772 \\
\hline Eos $/ \mathrm{mm}^{2}$ & $62.1(36.8-109.9)$ & $78.4(51.9-91.8)$ & 0.772 \\
\hline Ascending colon & $\mathrm{N}=18$ biopsies & $\mathrm{N}=10$ biopsies & \\
\hline Eos/hpf $0.196 \mathrm{~mm}^{2}$ & $18.5(7.7-26.8)$ & $13.5(12.8-17.5)$ & 0.719 \\
\hline Eos/hpf $0.306 \mathrm{~mm}^{2}$ & $28.9(12.0-41.8)$ & $21.1(19.9-27.3)$ & 0.719 \\
\hline $\mathrm{Eos} / \mathrm{mm}^{2}$ & $94.4(39.2-136.5)$ & $68.9(65.1-89.3)$ & 0.719 \\
\hline Transverse colon & $\mathrm{N}=27$ biopsies & $\mathrm{N}=17$ biopsies & \\
\hline Eos/hpf $0.196 \mathrm{~mm}^{2}$ & $12.8(7.1-18.9)$ & $13.0(8.0-19.0)$ & 0.941 \\
\hline Eos/hpf $0.306 \mathrm{~mm}^{2}$ & $20.0(11.0-29.9)$ & $20.3(12.5-29.7)$ & 0.941 \\
\hline $\mathrm{Eos} / \mathrm{mm}^{2}$ & $65.4(35.9-96.7)$ & $66.3(40.8-97.1)$ & 0.941 \\
\hline Descending colon & $\mathrm{N}=19$ biopsies & $\mathrm{N}=12$ biopsies & \\
\hline Eos/hpf $0.196 \mathrm{~mm}^{2}$ & $11.0(5.1-20.0)$ & $13.0(9.3-15.0)$ & 0.559 \\
\hline Eos/hpf $0.306 \mathrm{~mm}^{2}$ & $17.2(8.0-31.2)$ & $20.3(14.4-23.4)$ & 0.529 \\
\hline Eos $/ \mathrm{mm}^{2}$ & $56.1(26.1-102.0)$ & $66.3(47.2-76.5)$ & 0.529 \\
\hline Sigmoid & $\mathrm{N}=20$ biopsies & $\mathrm{N}=17$ biopsies & \\
\hline Eos/hpf $0.196 \mathrm{~mm}^{2}$ & $7.4(5.3-9.9)$ & $8.0(5.1-11.0)$ & 0.775 \\
\hline Eos/hpf $0.306 \mathrm{~mm}^{2}$ & $11.5(8.3-15.5)$ & $12.5(8.0-17.2)$ & 0.775 \\
\hline Eos $/ \mathrm{mm}^{2}$ & $37.6(26.9-50.5)$ & $40.8(26.1-56.1)$ & 0.775 \\
\hline Rectum & $\mathrm{N}=20$ biopsies & $\mathrm{N}=21$ biopsies & \\
\hline Eos/hpf $0.196 \mathrm{~mm}^{2}$ & $4.6(1.8-9.3)$ & $5.0(1.9-7.8)$ & 0.770 \\
\hline Eos/hpf $0.306 \mathrm{~mm}^{2}$ & $7.1(2.8-14.4)$ & $7.8(3.0-12.2)$ & 0.770 \\
\hline $\mathrm{Eos} / \mathrm{mm}^{2}$ & $23.3(8.9-47.2)$ & $25.5(9.8-40.0)$ & 0.770 \\
\hline
\end{tabular}

Eos/hpf, Eosinophils per high power field; IQR, interquartile range

the presence or not of abdominal pain, but may be considered in the context of the geographical variations that we found in all of the GI biopsies, a finding consistent with previous reports on eos concentrations in normal colonic mucosa [15]. The reasons for these geographical variations are not clear. Some studies have suggested that environmental aero- or food allergens may have an impact on GI tissue eosinophilia $[15,16]$, while others do not agree [17]. As, however, data on diet, drugs or history of atopy were not collected, we cannot delineate the impact of any of the above factors on the observed differences. Additionally, it cannot be excluded that the differences found between centers may be due to the different volumes of the samples collected in the participating centers, with Madrid and Rome having collected half and one third respectively, compared to Athens.

Furthermore, an important problem with existing reports in the literature on GI tissue eosinophilic infiltration is that the eos counts are expressed in cell number/hpf, although the size of the hpf is not specified. It is known, however, that the area of the hpf is strongly correlated with the technical parameters of the microscope, such as the magnification of the objective lens and the diameter of the ocular. The diversity among the technical parameters of commercially available microscopes may cause up to fivefold discrepancies in the eos counts of the same biopsy sample. To avoid heterogeneity, it has been suggested that eos 
should be counted in at least 5 fields of view from the area with peak eos density, and that eos density should be reported in cells $/ \mathrm{mm}^{2}$ [18], a methodology adopted in this study.

Interestingly, we found that the number of children fulfilling the Rome IV criteria for FGID diagnosis was almost twice that when the Rome III criteria were used. Other recent studies have also shown that Rome III criteria underestimated the number of children with FGIDs [10].

The association of FGIDs with the eosinophilic infiltration of GI tissue is controversial. Similarly to other studies in children [19], we were not able to demonstrate any differences in the eosinophilic infiltration of the GI tract of children diagnosed with FGIDs. In contrast, a recent meta-analysis of FD, including studies in adults and in children, reported a significant increase in eosinophilic density in both the gastric and duodenal mucosa of patients compared to healthy controls [20]. However, 3 of 4 studies performed in children with FD showed no differences in the eos counts in the gastric mucosa $[7,21,22]$, while the same was shown by other studies in the childhood duodenal mucosa [8] between patients and healthy controls. Furthermore, although the counts of eos in the GI mucosa of children with $\mathrm{FGID}^{+}$did not differ, we did find a greater incidence of other histological abnormalities, such as increased intraepithelial eos and eosinophilic cryptitis, that occur rarely in healthy GI tissue $[3,19,23]$. Based on the above, we can hypothesize that there may be a subtle inflammation in these patients, possibly related to an imbalance in GI homeostasis that may result from any number or combination of factors, including dysbiosis, loss of barrier integrity, genetic predisposition, or immune responses to dietary or luminal antigens [24]. The mild degranulation of eos-associated extracellular granules that we found toward the edge of the tissue sample biopsy in almost one third of the intestinal biopsies can be attributed to tissue trauma resulting from the use of forceps for obtaining the tissue, causing disruption of the eos cellular integrity [25].

Our study had several limitations. One was its retrospective design, which did not allow strict conclusions to be drawn regarding the geographical variations in the eosinophilic infiltration of the GI tract. As data on diet, drugs and history of allergy were not reported, the potential impact of any of these factors on the eosinophilic density of GI tissue cannot be excluded. It is also possible that the observed differences could be attributed to differences in the numbers of the biopsy samples collected by the participating centers, with Madrid and Rome having collected half and one third, respectively, of the number of biopsy samples collected in Athens. Furthermore, we did not assess inter-pathologist variation in assessing histology slides amongst the participating centers. It should be noted, however, that the criteria and the procedure for the histology assessment were discussed and agreed upon by all of the pathologists before the start of the study, while three quarters of the total number of histology slides (710 of 1014) were also compared and reviewed by a senior pathologist (KS), minimizing potential errors. In addition, it is not possible to draw conclusions from this study as to whether the eosinophilic infiltration of the duodenum was intracryptal or intravillous. As the aim of the study was to provide data on the peak counts of eos in the "healthy" GI mucosa, the pathologists focused on the eosinophilic "hot spots", e.g., places with the greatest eos concentration, without indicating their exact location (intracryptal or intravillous).

In conclusion, this study provides normative values on eosinophilic distribution and density in different segments of the GI tract of children with no organic diseases, which could be used in the evaluation of children with suspected EGIDs beyond the esophagus, the diagnosis of which in the absence of baseline data, is often a challenging issue, as it is based on the pathologist's subjective judgment.

\section{Summary Box}

\section{What is already known:}

- Eosinophilic gastrointestinal (GI) disorders (EGID) are rare, chronic inflammatory disorders of the GI tract, characterized by dense eosinophilic inflammation of the GI tract, in the clinical context. However, due to the absence of normative values on eosinophiic infitration of the healthy GI tract mucosa, the EGID diagnosis is often challenging as it relies on the pathologist's subjective judgment

- The geographical variations in the eosinophil distribution or eosinophilic density in the GI tract of children with no organic diseases have been poorly defined

- Other diseases, such as inflammatory bowel disease, have been associated with increased eosinophil counts in the GI mucosa, while controversial data exist on the impact of functional GI disorders (FGIDs) on the distribution and density of eosinophils in the childhood GI tract

\section{What the new findings are:}

- The reported in this study peak eosinophil counts in the epithelium of different segments of the GI tract of children with no organic diseases, provide normative values, which could be used for the evaluation of children with suspected EGID

- The shown in this study differences in the peak counts of eosinophils in the same segments of the GI tract are attributed to the different sizes of high power fields used for calculation by different pathologists. The above discrepancies highlight the importance of using eosinophil density (eos $\left./ \mathrm{m}^{2}\right)$ instead of eos/hpf, that is a universally accepted tool, independent of the size of hpf

- A geographical variation in GI tissue eosinophilic density was found, but the causative factors need further evaluation

- Eosinophil distribution and eosinophilic density in the GI tract do not differ in children with an FGID diagnosis, according to the Rome IV criteria, compared to those without such a diagnosis 


\section{References}

1. Aceves S, Hirano I, Furuta GT, Collins MH. Eosinophilic gastrointestinal diseases-clinically diverse and histopathologically confounding. Semin Immunopathol 2012;34:715-731.

2. Lowichik A, Weinberg AG. A quantitative evaluation of mucosal eosinophils in the pediatric gastrointestinal tract. Mod Pathol 1996;9:110-114.

3. DeBrosse CW, Case JW, Putnam PE, Collins MH, Rothenberg ME. Quantity and distribution of eosinophils in the gastrointestinal tract of children. Pediatr Dev Pathol 2006;9:210-218.

4. Saad AG. Normal quantity and distribution of mast cells and eosinophils in the pediatric colon. Pediatr Dev Pathol 2011;14:294-300.

5. Chernetsova E, Sullivan K, de Nanassy J, et al. Histologic analysis of eosinophils and mast cells of the gastrointestinal tract in healthy Canadian children. Hum Pathol 2016;54:55-63.

6. Lee EH, Yang HR, Lee HS. Quantitative analysis of distribution of the gastrointestinal tract eosinophils in childhood functional abdominal pain disorders. J Neurogastroenterol Motil 2018;24:614-627.

7. Wauters L, Nightingale S, Talley NJ, Sulaiman B, Walker MM. Functional dyspepsia is associated with duodenal eosinophilia in an Australian paediatric cohort. Aliment Pharmacol Ther 2017;45:1358-1364.

8. Lee EH, Yang HR, Lee HS. Analysis of gastric and duodenal eosinophils in children with abdominal pain related functional gastrointestinal disorders according to Rome III criteria. $J$ Neurogastroenterol Motil 2016;22:459-469.

9. Collins MH, Capocelli K, Yang G-Y. Eosinophilic gastrointestinal disorders pathology. Front Med (Lausanne) 2018;4:261.

10. Hyams JS, Di Lorenzo C, Saps M, Shulman RJ, Staiano A, van Tilburg M. Functional disorders: children and adolescents. Gastroenterology 2016;150:1456-1468.

11. Edwards T, Friesen C, Schurman JV. Classification of pediatric functional gastrointestinal disorders related to abdominal pain using Rome III vs. Rome IV criterions. BMC Gastroenterol 2018;18:41.

12. Rasquin A, Di Lorenzo C, Forbes D, et al. Childhood functional gastrointestinal disorders: child/adolescent. Gastroenterology 2006;130:1527-1537.

13. Strozzi D, Botacin MAS, Mekdessi MA, et al. Frequency of esophageal eosinophilia in a pediatric population from Central
Brazil. Sci Rep 2018;8:5000.

14. Kiss Z, Tél B, Farkas N, et al. Eosinophil counts in the small intestine and colon of children without apparent gastrointestinal disease: a meta-analysis. J Pediatr Gastroenterol Nutr 2018;67:6-12.

15. Pascal RR, Gramlich TL, Parker KM, Gansler TS. Geographic variations in eosinophil concentration in normal colonic mucosa. Mod Pathol 1997;10:363-365.

16. Mehta P, Furuta GT. Eosinophils in gastrointestinal disorders: eosinophilic gastrointestinal diseases, celiac disease, inflammatory bowel diseases, and parasitic infections. Immunol Allergy Clin North Am 2015;35:413-437.

17. Matsushita T, Maruyama R, Ishikawa N, et al. The number and distribution of eosinophils in the adult human gastrointestinal tract: a study and comparison of racial and environmental factors. Am J Surg Pathol 2015;39:521-527.

18. Saffari H, Peterson KA, Fang JC, Teman C, Gleich GJ, Pease LF 3rd. Patchy eosinophil distributions in an esophagectomy specimen from a patient with eosinophilic esophagitis: Implications for endoscopic biopsy. J Allergy Clin Immunol 2012;130:798-800.

19. Silva J, Canão P, Espinheira MC, Trindade E, Carneiro F, Dias JA. Eosinophils in the gastrointestinal tract: how much is normal? Virchows Arch 2018;473:313-320.

20. Du L, Chen B, Kim JJ, Chen X, Dai N. Micro-inflammation in functional dyspepsia: A systematic review and meta-analysis. Neurogastroenterol Motil 2018;30:e13304.

21. Ashorn M, Ruuska T, Karikoski R, Välipakka J, Mäki M. Gastric mucosal cell densities in Helicobacter pylori-positive and -negative dyspeptic children and healthy controls. J Pediatr Gastroenterol Nutr 1994;18:146-151.

22. Faure C, Patey N, Gauthier C, Brooks EM, Mawe GM. Serotonin signaling is altered in irritable bowel syndrome with diarrhea but not in functional dyspepsia in pediatric age patients. Gastroenterology 2010;139:249-258.

23. Elizabeth A. Montgomery. Biopsy interpretation of the gastrointestinal tract mucosa. In: Lynsandra Voltaggio (editor), third edition. Philadelphia: Wolters Kluwer; 2018, pp. 228-229.

24. Kato M, Kephart GM, Talley NJ, et al. Eosinophil infiltration and degranulation in normal human tissue. Anat Rec 1998;252:418-425.

25. Burns G, Pryor J, Holtmann G, Walker MM, Talley NJ, Keely S. Immune activation in functional gastrointestinal disorders. Gastroenterol Hepatol (N Y) 2019;15:539-548. 